\title{
Quality of HIV Counselling in South Africa
}

Mohlabane, N.; Peltzer, K., Mwisongo, A., Ntsepe, Y., Tutshana, B., Van Rooyen, H. and Knight, L.

\begin{abstract}
HIV counselling and testing (HCT) has become increasingly available in South Africa since the 1990s. Over 4500 public health facilities offer client and provider-initiated HIV counselling and testing. Counselling and testing remain key components of HIV/AIDS prevention as they provide an entry point into prevention, care, treatment and support services. This paper examines the quality of HIV counselling in government and Nongovernmental (NGO) facilities and reviews adherence to the HCT policy guidelines during counselling sessions in $67 \mathrm{HIV}$ counselling and testing (HCT) sites across 8 South African provinces. The assessment used both quantitative and qualitative methods. In total 149 structured observations of counselling sessions were conducted using a written checklist and audio recording. This assessment confirms that while counselling does occur prior and post HIV testing, the quality of counselling differs between sites and does not match the South African HCT policy guidelines. The following key aspects were not adequately discussed with clients: risk assessment and reduction, partner involvement, supportive care and treatment for those testing HIV positive. Confidentiality was also compromised by frequent interruptions during some sessions. The assessment indicated that ongoing mentoring of counsellors needs to be addressed, to make the HCT programme more effective.
\end{abstract}

\section{Introduction}

Counselling and testing remain key components of HIV/AIDS prevention as they provide an entry point into prevention, care, treatment and support services (Kipitu 2005; Wanyenze et al. 2008; Grabbe et al. 2010). During the counselling and testing process, an individual undergoes confidential counselling to enable them to make an informed choice about learning their HIV status and to take appropriate action (Haraka et al. 2012). Pre-test counselling aims to ex- plain the HIV testing process, giving individuals relevant HIV information by assessing HIV knowledge, correcting misconceptions about HIV and assessing as well as reviewing the client's risk reduction options in order to motivate behaviour change (Magongo et al. 2002; Njeru et al. 2011). Post-test counselling on the other hand, assesses the client's readiness to receive their HIV results, discusses the meaning of test results and assesses the impact of the results on the client (Magongo et al. 2002). The manner in which clients are encouraged into taking the HIV test and receive their HIV test results plays a key role in their future HIV prevention behaviour for both HIV positive and HIV negative clients. However, counsellors do not seem to be consistently applying a standard counselling approach (Setswe 2009; Dewing et al. 2012; Mpiima et al. 2013). Counsellors at various set- tings have been observed 
and reported to be prone to giving information and advice instead of exploring personalised alternatives to managing client situations (Dewing et al. 2013). Also that literature looking at HIV counselling in the Southern Africa region focus on service-delivery approaches that bring clients into HIV counselling and testing (HCT) instead of the quality of counselling during the sessions (Family Health International 2005; Setswe 2009; Nation- al Department of Health South African HCT Guidelines 2010). These service-delivery models include the providerinitiated-counselling and testing (PICT), HCT at special events or campaigns, HCT at workplaces and at special places such as prisons.

The quality of HIV counselling and testing is critical in ensuring the potential impact of risk reduction (Sherr et al. 2007). The current pre- dominant counselling models such as the Egan model in South Africa and the TASO model in Uganda are seen as less structured to effect any behavioural change compared to the risk reduction model used in many other parts of the world (Van Rooyen et al. 2010). The Egan model, for example, is described as client-centred and is used by skilled helpers, is collaborative and its main task is to assist the client to explore personalised alternatives to their situation (Van Rooyen et al. 2010; Dewing et al. 2012). In a study evaluating the standard of care during ART adherence counselling in the Western Cape, South Africa, it was established that while counsellors described their counselling approach as having the Egan's client-centred principle, the actual observations of the counselling sessions did not evidence the practice (Dewing et al. 2012). Both the Egan and the TASO models are problem solving oriented with a focus on helping the client state why they have come in for HIV testing and consider their options regarding testing as well as helping them come up with a plan regarding the HIV test. Additionally, the AIDS Support Organisation (TASO) approach also tends to focus on HIV positive clients to adopt the Abstain, Be faithful and Condom-use (ABC) approach. The limitation of the TASO approach however is that while sick clients may adhere to the $\mathrm{ABC}$ principles, when they recover and their sexual desires resurface; their adherence to the $\mathrm{ABC}$ principles is reduced (Mpiima et al. 2013). The risk reduction counselling model on the other hand consists of brief, collaborative, patient-centred discussions between a trained lay counsellor and the HIV patient during routine clinical visits. The counsellor uses Motivational Interviewing techniques to assess the patient's sexual risk behaviour, identify specific Information-Motivation-Behavioral (IMB) barriers that may be preventing the patient from consistently engaging in safer sex as well as explore possible strategies that the patient can use to address his/her specific barriers and then negotiate an individually tailored and achievable behavioural change goal with the patient for the following visit (Cornman et al. 2011).

An effective counselling intervention is required for the achievement of behavioural change that promote HIV prevention. Interactive counselling approaches are structured and focus on addressing the emotional and psycho- social concerns of the patient and motivate them into changing their behaviour (Strode et al. 2005; 
Haffejee et al. 2010; Cornman et al. 2011; Kaaya et al. 2013). Such counselling interventions are preferred and relevant compared to the didactic, informational approaches. However, in the South African context, while there have been efforts to train counsellors and supervisors at district, provincial and national level on the use of the risk reduction model, this model has not been widely implemented (Van Rooyen et al. 2010). The counselling model used and the quality of the service can thus have an impact on future prevention strategies.

HIV counselling and testing have become increasingly available in South Africa since the 1990s, over 4500 public health facilities offer client- initiated HIV counselling and testing (CICT) and provider-initiated counselling and testing (PICT). HIV counselling and testing (HCT) is offered through mobile services as well as com- munity-based models of non-governmental, faith and communitybased organisations. The 2014 survey of HIV prevalence in the country reported an increase in HCT uptake to 65.5 percent (Shisana et al. 2014); while the results from earlier surveys conducted in 2002 and 2005 showed that HIV testing increased from 18.9 percent to 39.3 percent. By 2008 HCT had increased to 50 percent (Shisana et al. 2009). In 2003, the National Department of Health (NDOH) developed HIV counselling and testing guidelines that are based on international standards, including those developed by the World Health Organisation (WHO) as well as the Centers for Disease Control and Prevention (CDC). These guidelines have been reviewed and updated based on recommendations by WHO and CDC. They cater for both the public and private sector and ad- dress issues pertaining to HIV counselling and testing as well as ensure that services are standardized throughout the country $(\mathrm{NDOH}$ 2010). A recent qualitative assessment of HIV counselling and testing by MacCarthy et al. (2014) in Salvador, Brazil among pregnant women, revealed that services occur with insufficient and inconsistent attention to the three Cs; counsel- ling, consent and confidentiality and therefore cannot be characterised as quality services. However, the findings were not triangulated with additional data and only relied on participant's accounts. A South African study evaluating voluntary counselling and testing (VCT) services for mine workers, established that counsel- ling was in this context also poorly and inconsistently provided. The study found that community volunteers were inadequately equipped with counselling skills in their training and they failed to address the window period in pre-test counselling (Ginwalla et al. 2002). This finding is similar to a study conducted in Kenya, which reported that window period, partner involvement and follow-up support were haphazardly dealt with during HIV counselling (Delva et al.

2006).

Quality is defined as the consistent delivery of a product or service, according to expected standards (Dinku and Andargie 2013). According to Zachariah et al. (2009), there is evidence that inappropriate curricula and poor supervision affects the quality of care provided by health workers and suggests that in HIV counselling, 
quality does determine outcome. Quality counselling is argued to increase the likelihood that a person diagnosed with HIV will adhere to preventative guidelines for HIV and will cope better with his or her diagnosis (Mwamburi et al. 2005). Richards and Marquez (2005) cite various structural challenges faced by lay counsellors; including inadequate training which leaves them ill-equipped to address issues such as serodiscordancy and couple counselling during sessions. In addition, counsellors often lack the skills to deal with crises and are ineffective in helping HIV-positive individuals cope with their results (Mwamburi et al. 2005). Haffejee et al. (2011) found that due to lack of training; counsellors failed to pay attention to symptoms of mental illness, although patients testing HIV positive are highly likely to suffer mental distress.

Assessment of the quality of HIV counsel- ling in South Africa has also been poorly ad- dressed. Chopra et al. (2005) conducted an assessment of HIV counselling in the context of prevention of mother to child transmission (PMTCT) but only focused on three pilot sites and hence do not provide a general picture of counselling services in the country. Similarly, Haffejee et al. (2011) conducted a baseline study to assess the counselling and support services available to people living with HIV (PLWH) in one province of South Africa. Both studies cited programmatic and structural constraints that comprised the quality of counselling. There is a lack of information in the literature on recent assessments of the quality of HIV counselling among the general South African population. This paper thus examines the quality of HIV counselling and the process followed during counselling sessions in 67 HCT sites in eight provinces of South Africa.

\section{Objectives}

- To compare the quality of HIV counselling between government and NGO facilities

- To assess counsellors adherence to the HCT policy guidelines during counselling sessions

- To make recommendations based on the findings

\section{METHODOLOGY}

\section{Study Setting and Design}

This analysis is based on data from a rapid appraisal of 67 government and NGO facilities that provide HCT services in eight provinces of South Africa. One province was excluded since permission to conduct the study could not be obtained. All the sites were assessed during site visits and through observations of counselling and testing sessions. The assessment used both quantitative and qualitative methods which enabled the assessment of the quality of counsel- ling using different techniques.

\section{Sampling and Procedures}

The quality and content of counselling were assessed at each of the HCT sites through in- person observation of two to three pre and post con sent was obtained from both the counsellor and the client prior to observation and the counselling

$$
\text { https://repository.uwc.ac.za/ }
$$


session was audio-recorded. The consent process was brief and the observer was as unobtrusive as possible to minimise the impact on the counselling session. In addition, counselling sessions were conducted in the local language and all observers were fluent in several languages that were spoken in the province they were allocated in. Observers had post-graduate qualifications and were trained in counselling.

An observation checklist was designed to assess the minimum standards for quality HIV counselling in group information, pre and post-test counselling sessions. The checklist assessed the content of the sessions and the skill with which the counselling was delivered. A score was allocated for the ability of the counsellor to gather information from the client, provide them with correct and complete information, discuss their personal circumstances, deal with partner notification, reinforce prevention messages and adequately refer to care. In addition, they were assessed on whether they had good interpersonal skills and were able to provide the client with relevant support. The checklist was based on the 2010 National HCT guidelines (National Department of Health South African HCT Guidelines 2010) which outline key aspects to be included in the group information, pre and post- test counselling sessions as summarised below:

\section{Group Information}

HIV acquisition and transmission, effective HIV prevention measures, the importance and advantages of early HIV testing, information about the HIV testing process, confidentiality and shared confidentiality, discussion on the option not to take the test or to test later, the importance of TB symptomatic screening, and referral to HIV and AIDS related services.

\section{Pre-test Counselling}

Opportunity to respond to unanswered questions and clarifying misunderstandings, discussion of specific issues of individual and assessment of individual risk, and risk reduction and the window period should the client test HIV negative, discussion of HIV prevention strategies, follow-up medical care should the client test HIV positive, discussion on partner involvement and referral for testing, option to refuse testing and obtaining written or verbal informed consent for HIV testing.

\section{Post-test Counselling Session}

HIV-negative clients should be offered a comprehensive post-test counselling prevention package that includes information and advantages of Medical Male Circumcision (MMC), TB screening, risk reduction, correct and regular use of condoms. They should be encouraged to repeat the test three months after exposure to exclude the possibility of the window period. Window period should be explained.

HIV-positive clients must be given their test results and counselled post-test about their HIV status only after the second confirmatory test is also positive. Clients should be informed and counselled about possible emotional responses and what 
impact these emotions can have on adherence to healthy lifestyle choices. These clients also need comprehensive information on how to reduce the risk of transmission, ongoing, positive living, healthy lifestyles and nutrition and ongoing referral for psychosocial sup- port (for example, support groups), preventive package including correct regular use of condoms, and medical services when needed. After post-test counselling all HIV-positive clients must be referred for laboratory staging by CD4 count and clinical staging by a clinician trained in HIV and AIDS clinical management. They must also be screened for symptomatic TB signs and referred for diagnosis if suspected and either prepared for ART or referred to attend the well- ness services provided (pre ART management) if they do not qualify for ART immediately (National Department of Health South African HCT Guidelines 2010).

\section{DataAnalysis}

Recorded qualitative data gathered from the counselling session observations was transcribed and translated into English. Data were analysed using grounded theory techniques (Miles and Huberman 1994) and collaboratively coded and reviewed. Major trends and cross cutting themes were identified and issues for further exploration were prioritized for final analysis. All quantitative data from the observation of two to three pre and post-test counselling sessions. Written informed con- sent was obtained from both the counsellor and the client prior to observation and the counsel- ling session was audio-recorded. The consent process was brief and the observer was as un- obtrusive as possible to minimise the impact on the counselling session. In addition, counsel- ling sessions were conducted in the local language and all observers were fluent in several languages that were spoken in the province they were allocated in. Observers had post-graduate qualifications and were trained in counselling.

An observation checklist was designed to assess the minimum standards for quality HIV counselling in group information, pre and post- test counselling sessions. The checklist assessed the content of the sessions and the skill with which the counselling was delivered. A score was allocated for the ability of the counsellor to gather information from the client, provide them with correct and complete information, discuss their personal circumstances, deal with partner notification, reinforce prevention messages and adequately refer to care. In addition, they were assessed on whether they had good interpersonal skills and were able to provide the client with relevant support. The checklist was based on the 2010 National HCT guidelines (National Department of Health South African HCT Guidelines 2010) which outline key aspects to be included in the group information, pre and post- test counselling sessions as summarised below:

\section{Group Information}

HIV acquisition and transmission, effective HIV prevention measures, the importance and advantages of early HIV testing, information about the HIV testing process, confidentiality and shared confidentiality, discussion on the 
option not to take the test or to test later, the importance of TB symptomatic screening, and referral to HIV and AIDS related services.

\section{Pre-test Counselling}

Opportunity to respond to unanswered questions and clarifying misunderstandings, discussion of specific issues of individual and assessment of individual risk, and risk reduction and the window period should the client test HIV negative, discussion of HIV prevention strategies, follow-up medical care should the client test HIV positive, discussion on partner involvement and referral for testing, option to refuse testing and obtaining written or verbal informed consent for HIV testing.

\section{Post-test Counselling Session}

$H I V$-negative clients should be offered a comprehensive post-test counselling prevention package that includes information and advantages of Medical Male Circumcision (MMC), TB screening, risk reduction, correct and regular use of condoms. They should be encouraged to repeat the test three months after exposure to exclude the possibility of the window period. Window period should be explained.

$H I V$-positive clients must be given their test results and counselled post-test about their HIV status only after the second confirmatory test is also positive. Clients should be informed and counselled about possible emotional responses and what impact these emotions can have on adherence to healthy lifestyle choices. These clients also need comprehensive information on how to reduce the risk of transmission, ongoing, positive living, healthy lifestyles and nutrition and ongoing referral for psychosocial sup- port (for example, support groups), preventive package including correct regular use of condoms, and medical services when needed. After post-test counselling all HIV-positive clients must be referred for laboratory staging by CD4 count and clinical staging by a clinician trained in HIV and AIDS clinical management. They must also be screened for symptomatic TB signs and referred for diagnosis if suspected and either prepared for ART or referred to attend the well- ness services provided (pre ART management) if they do not qualify for ART immediately (National Department of Health South African HCT Guidelines 2010).

\section{Data Analysis}

Recorded qualitative data gathered from the counselling session observations was transcribed and translated into English. Data were analysed using grounded theory techniques (Miles and Huberman 1994) and collaboratively coded and reviewed. Major trends and cross cutting themes were identified and issues for further exploration were prioritized for final analysis. All quantitative data from the observation checklists were analysed using cross tabulations and basic descriptive statistics in the Statistical Package for Social Scientists (SPSS, Version 20.0).

\section{Ethical Aspects}

Ethical approval was obtained from the Hu- man Sciences Research Council (HSRC) 
Ethics Committee and the Centers for Disease Control (CDC). The South African National Department of Health, eight of nine Provincial Departments of Health and the individual HCT sites granted permission prior to conducting the study. Written informed consent was obtained from all participants prior to the interviews.

\section{Results}

In all, $73 \mathrm{HCT}$ sites were approached for inclusion in the study; 67 agreed and 6 refused to participate (all NGOs), giving a response rate of 91.8 percent. Table 1 shows the number and type of sites that were visited as well as the type of sessions that were observed by site. In total one hundred and forty nine; 9 group and 140 pre and post-test counselling sessions were observed. A total of 106 (71\%) and 39 (26.2\%) sessions were observed in government and NGO facilities respectively, whilst the remainder were in both private and workplace facilities. The majority of the sessions was observed in rural areas 68 (45.9\%), followed by peri-urban 39 (26.2\%) and urban 34 (23.0\%) facilities. The majority of the sessions was conducted by lay counsellors

\section{Pre-test Counselling}

\section{Information Giving}

The content assessment of the pre-test counselling session (Table 2) revealed that during 89 (87.3\%) Government and 30 (78.9\%) NGO sessions, clients' HIV knowledge was explored. During $83(81.4 \%)$ government and 29 (76.3\%) NGO sessions counsellors clarified clients' misunderstanding about HIV issues. The HIV testing process as well as window period was also discussed during 80 (78.4\%) government and $29(76.3 \%)$ NGO sessions that were observed. The clients option to refuse or postpone HIV testing

\begin{tabular}{|c|c|c|c|c|c|c|c|}
\hline & \multicolumn{2}{|c|}{$\begin{array}{l}\text { Government } \\
n(\%)\end{array}$} & \multicolumn{2}{|c|}{$\begin{array}{l}\text { NGOs } \\
n(\%)\end{array}$} & $\begin{array}{l}\text { Work place } \\
n(\%)\end{array}$ & $\begin{array}{r}\text { Private } \\
n(\%)\end{array}$ & $\begin{array}{l}\text { Over all } \\
n(\%)\end{array}$ \\
\hline & \multicolumn{2}{|c|}{$106(71.1)$} & \multicolumn{2}{|c|}{$39(26.2)$} & $2(1.3)$ & $2(1.3)$ & $149(100)$ \\
\hline $\begin{array}{l}\text { 1ype of Area } \\
\text { Urban area }\end{array}$ & \multirow{4}{*}{\multicolumn{2}{|c|}{$\begin{array}{r}21(61.8) \\
20(52.6) \\
3(37.5) \\
62(91.2)\end{array}$}} & \multicolumn{2}{|c|}{$11(32.4)$} & 0 & $2(5.9)$ & $34(23.0)$ \\
\hline Peri-urban area & & & \multicolumn{2}{|c|}{$17(43.6)$} & $2(5.3)$ & 0 & $39(26.2)$ \\
\hline Informal settlement & & & \multirow{2}{*}{\multicolumn{2}{|c|}{$\begin{array}{l}5(62.5) \\
6(8.8)\end{array}$}} & 0 & 0 & $8 \quad(5.4)$ \\
\hline Rural area & & & & & 0 & 0 & $68(45.9)$ \\
\hline $\begin{array}{l}\text { Type of Counselling Session } \\
\text { Group }\end{array}$ & & \multicolumn{2}{|l|}{7} & & & 9 \\
\hline $\begin{array}{l}\text { Group } \\
\text { Pre and post-test }\end{array}$ & \multicolumn{2}{|l|}{$104^{2}$} & \multicolumn{2}{|l|}{32} & 2 & $\begin{array}{l}0 \\
2\end{array}$ & 140 \\
\hline \multirow[t]{2}{*}{ Pre-test counselling $(n=140)$} & & \multicolumn{3}{|c|}{ Government $(n=102)$} & \multicolumn{2}{|c|}{$N G O(n=38)$} & \multirow{2}{*}{$\begin{array}{r}\text { Chi-square } \\
\text { p-value }\end{array}$} \\
\hline & & $N$ & & $\%$ (sessions) & $N$ & $\%$ (sessions) & \\
\hline HIV knowledge & & 89 & & 87.3 & 30 & 78.9 & 0.322 \\
\hline Clarify misunderstanding & & 83 & & 81.4 & 29 & 76.3 & 0.611 \\
\hline Confidentiality & & 74 & & 72.5 & 32 & 84.2 & 0.331 \\
\hline Individual risk assessment & & 50 & & 49.0 & 24 & 63.2 & 0.293 \\
\hline HIV testing process & & 80 & & 78.4 & 29 & 76.3 & 0.612 \\
\hline HIV prevention strategies & & 86 & & 84.3 & 28 & 73.3 & 0.158 \\
\hline Follow-up and management & & 79 & & 77.5 & 25 & 65.8 & 0.186 \\
\hline Partner involvement & & 65 & & 63.7 & 25 & 65.8 & 0.683 \\
\hline Opt out & & 45 & & 44.1 & 25 & 65.8 & 0.062 \\
\hline Informed consent & & 94 & & 92.2 & 38 & 100.0 & 0.206 \\
\hline
\end{tabular}

was discussed during only 45 (44\%) government and 25 (65.8\%) NGO sessions. 
Fewer than half 50 (49\%) of sessions conducted in government facilities included individual risk assessment of clients compared to 24 (63.2\%) sessions conducted in NGO facilities. Although during the majority of the sessions, 84 percent government and 73.7 percent NGO, HIV prevention strategies such as condom use were discussed with clients, however partner involvement and referral for testing was only discussed in 63.7 and 65.8 percent government and NGO sessions respectively.

\section{Confidentiality and Privacy}

Confidentiality has been regarded as an important factor to be considered in HCT. It was mentioned in a higher proportion of sessions conducted in NGO facilities (84.2\%) compared to government facilities (72.5\%). From the session recordings and observations, it was evident that in NGO facilities the counsellor followed a written guideline when conducting the counselling session, this seemed to assist the counsellor to ensure that all the necessary in- formation was discussed. In almost all sessions observed in NGO facilities, confidentiality was discussed with clients. The explanation was clear and simple and also included the fact that disclosure of results is voluntary.

'Again, I'm going to discuss our confidentiality, everything that we say in this room will remain between the three of us and I assure you no one will know what we are doing in here unless you decide that you want to disclose your status to your family or friends'(Male Counsellor, NGO, Limpopo).

During the counselling observations, it was evident in some instances that the counselling environment is not private or used exclusively for counselling. In some government facilities, counselling rooms were also used as storage rooms. Interruptions by other facility staff could be picked up during the actual session from the recordings and observations.

\section{Informed Consent}

The content assessment revealed that this key aspect was discussed during the majority of the counselling sessions observed in both government (92.2\%) and NGO facilities (100\%).

This finding was also evident from the session recording, a counsellor was able to explain the importance of testing early and knowing ones HIV status as well as the follow up care if one tests HIV positive: 'and again I can say that when a person is positive or maybe you find yourself that you are sick that doesn't mean is the end of the life but at the end is that it is important for a person to know his/her status because many people end up knowing they have the virus when it's too late and there is no more turning back to sort everything but it is important for people to get tested and know their status, if ever you find that you are positive, we will (do)the procedure of checking your CD4 count and if it is below 
350 it is where you are going to start with your treatment (ARVs) and if is above that number they are going to give the pills that will boost your body...'(Male Counsellor, NGO, Limpopo).

\section{HIV Testing-focused Approach}

During some counselling sessions it was evident from the recordings that some counsellors focused only on the testing process with- out discussing any other issues pertaining to HIV such as transmission and prevention. Additionally, other aspects pertaining to pre-test counselling such as risk assessment were not discussed with clients. The counsellors seemed comfortable conveying information about the test kits, the testing process and how the results will be read. This was observed in both NGO and government health facilities. Below is a quote from the session recording during pre-test counsel- ling where only the testing process was explained to the client and no other information pertinent to pre-test counselling was given.

"This is our test kit, we call this a chase buffer which is what we use to test. We prick you first and we add the buffer. We will wait for 20 minutes to get your test results. Are we clear?' (Female Counsellor, Government Facility, KwaZulu-Natal). The focus on testing information during counselling rather than HIV transmission, prevention, opportunistic infections such as TB, and STI was more prevalent in government facilities across all the eight provinces compared to NGOs.

\section{Post-test Counselling Communication of HIV Results}

Table 3 highlights the findings from the con- tent assessment of the post-test counselling sessions. During almost all the sessions, results were reported in a clear and simple manner to clients in both government and NGO facilities. Furthermore, the counsellors allowed adequate time for clients to learn their results and made sure clients understood the meaning of the test result. From the session recordings it was evi- dent that some sessions were very short and lacked important information; most counsellors gave the clients information about what the test kit would look like when an HIV negative or positive result is detected. In addition, some counsellors read the results to their clients while others asked the clients to read the results them- selves. In some instances, counsellors did not give clients information regarding the actual implications and importance of their results, in- stead they only told the client that 'you are negative'. In the instance where the counsellor showed the client the test kit to actually read and report the results to the counsellor, the client simply replied 'It says I have nothing'. This statement is ambiguous and to the client it implies that they are negative. This was also observed with HIV positive results where a client had to read their own results 'I can see one bright line and the second line is not clear...that the virus is there'. Some counsellors provided ambiguous results statements such as the example below: 
'If it was two..., this is positive, meaning that if you are told that you are negative be- cause you don't know what negative means but this is positive' (Male Counsellor, Government Facility, Mpumalanga).

These statements can be very confusing to clients because the counsellor is not saying whether the results are negative or positive.

\section{HIV Risk Reduction}

The content assessment revealed that, risk reduction strategies were reviewed during most sessions with a higher proportion of these being conducted in NGO (81.6\%) compared to government (74.5\%) facilities. Similarly, assessment of most recent exposure to HIV and information about the window period was discussed during 78.9 percent of NGO sessions compared to 65.7 percent of government facility sessions. However, the analysis revealed that there are no statistically significant differences between these findings by facility type.

From the counselling session recordings, it was evident that counsellors struggled to dis- cuss and guide clients to develop a risk reduction plan collaboratively instead they instructed clients to adopt specific HIV prevention strategies. Additionally, some counsellors struggled to provide factual information regarding the "window period' as reflected in the extract below, whereby a counsellor was giving a client HIV negative results and providing risk reduction information.

'It means you should continue using condoms or abstain until you come back in three months' time, we call this a window period..' (Female counsellor, NGO, Gauteng).

And 'You need to use a condom every time you have sex because men are not trustworthy. They cheat and do not use condoms.. Tell him that, they said in the clinic I need to use a condom every time I have sex' (Female Counsellor, Government Facility, Kwazulu Natal)

\section{Post-test Counselling: HIV Positive Results}

Table 4 highlights the findings of the con- tent assessment for the eighteen (12.5\%) post- test counselling sessions for HIV positive results. The counsellors dealt with immediate emotions in all the sessions in both government and

Table 3: Post-test counselling content assessment

\begin{tabular}{|c|c|c|c|c|c|}
\hline \multirow[t]{2}{*}{ Pre-test counselling $\quad(n=140)$} & \multicolumn{2}{|c|}{ Government $(n=102)$} & \multicolumn{2}{|c|}{$N G O(n=38)$} & \multirow{2}{*}{$\begin{array}{r}\text { Chi-square } \\
\text { p-value }\end{array}$} \\
\hline & $N$ & $\%$ (sessions) & $N$ & $\%$ (sessions) & \\
\hline Clear simple results & 100 & 98.0 & 38 & 100 & 0.385 \\
\hline Time allowed for results to sink in & 89 & 87.3 & 34 & 89.5 & 0.721 \\
\hline Checked understanding of results & 97 & 95.1 & 33 & 86.8 & 0.092 \\
\hline Checked meaning of result & 94 & 92.2 & 35 & 92.1 & 0.992 \\
\hline Review risk reduction strategies & 76 & 74.5 & 31 & 81.6 & 0.488 \\
\hline Window period & 67 & 65.7 & 30 & 78.9 & 0.298 \\
\hline Provide IEC material and condoms & 38 & 37.3 & 22 & 59.5 & 0.063 \\
\hline
\end{tabular}


Table 4: Post-test counselling content assessment: HIV positive results

\begin{tabular}{|c|c|c|c|c|c|}
\hline \multirow{2}{*}{$\begin{array}{l}\text { Pre-test counselling }(n=140) \\
\text { (HIV positive results } n=18)\end{array}$} & \multicolumn{2}{|c|}{ Government $(n=15)$} & \multicolumn{2}{|c|}{$N G O(n=3)$} & \multirow{2}{*}{$\begin{array}{c}\text { Chi-square } \\
\text { p-value }\end{array}$} \\
\hline & $N$ & $\%$ (sessions) & $N$ & $\%$ (sessions) & \\
\hline Dealt with immediate emotions & 15 & 100.0 & 3 & 100.0 & 0.461 \\
\hline Discussed implications of results & 13 & 86.7 & 2 & 66.7 & 0.536 \\
\hline Disclosure & 13 & 86.7 & 1 & 33.3 & 0.389 \\
\hline Assessed support systems & 10 & 66.7 & 3 & 100.0 & 0.365 \\
\hline Follow-up care and support & 14 & 93.3 & 1 & 33.3 & 0.655 \\
\hline Identified options and resources & 9 & 60.0 & 3 & 100.0 & 0.635 \\
\hline Risk reduction plan & 11 & 73.3 & 3 & 100.0 & 0.389 \\
\hline Partner referral & 11 & 73.3 & 3 & 100.0 & 0.271 \\
\hline Family planning & 11 & 73.3 & 2 & 66.7 & 0.628 \\
\hline Immediate plans & 11 & 73.3 & 2 & 66.7 & 0.628 \\
\hline Referral for medical care & 14 & 93.3 & 1 & 33.3 & 0.050 \\
\hline
\end{tabular}

NGO facilities. The session recording also con- firmed this finding as seen in the example be- low where a counsellor was providing post- test counselling for a client who tested HIV positive 'I am happy that you have accepted your status and you are right, many people are infected. Please avoid getting stressed by thinking about your status. It is important that you use a condom when you have sex with your partner..'(Female Counsellor, Government Facility, KwaZulu-Natal).

The content assessment revealed that discussion of implications of the HIV-positive result as well as follow-up care and support were observed during most sessions. This is an ex- ample from the session recordings of a counsellor giving a client who tested HIV positive information about follow-up and medical care' Today, we will need to take your blood sample so that we can check your CD4 count, the soldiers of the body. If your $\mathrm{CD}_{4}$ cell count results are 350 and below, you will need to get enrolled for ARVs' (Female Counsellor, Government Facility, KwaZulu-Natal).

\section{Disclosure}

Disclosure of results was discussed during 86.7 percent of HIV-positive results in government compared to 33.3 percent NGO facilities. From the session recordings, it was evident that in some instances, when giving HIV positive results counsellors only provided information about disclosure and excluded all other important information such follow-up care and sup- port as well as partner referral as seen in the example below:

It is very important to disclose, there should at least be one person you disclose to for an example if you do not disclose it will be difficult to take treatment, like if you have a reminder in your phone then it goes off you will always switch off the phone because you did not disclose. But if you disclose the person you've disclosed to can support you.' (Female Counsellor, Government Facility, Free State)

\section{Quality of Counselling Skills}

The counselling skills were also assessed during the session observations; these were scored 1 to 3 ; 1 being 'needs improvement' 2 'satisfactory' and 3 'done well'. Table 5 shows the results of the counselling skills assessment for those who scored 3 . 
Greeting and introduction of self to clients was observed during a higher proportion of sessions observed at NGO (84.6\%) compared to government facilities (70.6\%). Although 'active listening' and showing 'supportive attitude' were observed during a few sessions in both NGO and government facilities, majority were observed during sessions conducted in NGO facilities. The results also highlight that basic counselling skills such as summarizing, probing, using silence appropriately and questioning were poorly conducted in both government and NGO facilities.

\section{Discussion}

The findings of this study highlight that there are several gaps pertaining to the content of the HIV counselling sessions observed; there was transgression from the HCT policy guide- lines whereby lay counsellors were not discussing some key aspects as specified in the guidelines. This indicates the need for development

\begin{tabular}{|c|c|c|c|c|}
\hline \multirow[b]{2}{*}{ Counselling skills } & \multicolumn{2}{|c|}{ Government } & \multicolumn{2}{|c|}{$N G O$} \\
\hline & $N$ & $\%$ (sessions) & $N$ & $\%$ (sessions) \\
\hline Greeting & 72 & 70.6 & 33 & 84.6 \\
\hline Introduction & 71 & 69.6 & 32 & 82.1 \\
\hline Active listening & 49 & 48.0 & 21 & 53.8 \\
\hline Supportive and non-judgemental & 59 & 57.8 & 27 & 69.2 \\
\hline Open and closed ended questions used appropriately & 33 & 32.4 & 12 & 30.8 \\
\hline Clarification & 31 & 30.4 & 13 & 33.3 \\
\hline Probing & 33 & 32.4 & 13 & 33.3 \\
\hline Summarizing & 39 & 38.2 & 17 & 43.6 \\
\hline Current HIV information & 37 & 36.3 & 21 & 55.3 \\
\hline Accommodate language difficulty & 79 & 77.5 & 29 & 78.4 \\
\hline
\end{tabular}

and implementation of a quality improvement strategy across HCT sites to address the identified gaps. Chopra et al. (2005) reported similar findings in their analysis of VCT sessions for PMTCT counselling in South Africa. Although the sample size was small and findings cannot be generalised to HIV counselling services in the country as a whole nor to PMTCT in particular, they did however provide an indication of the prevailing situation at the time the study was conducted. A recent qualitative assessment by MacCarthy et al. (2014) in Brazil also high- lighted that quality of counselling provided to pregnant women was compromised. The occurance of the three Cs; pre and post-test counsel- ling, confidentiality and informed consent was inconsistent among the research participants.

Pre-test counselling gives individuals an opportunity to receive relevant information, correct misconceptions about HIV, and assess their risk so as to motivate for behaviour change if necessary (Njeru et al. 2011). Nevertheless, the content assessment revealed that although key aspects such as assessment of HIV knowledge, the testing process, informed consent and HIV prevention strategies were discussed in the majority of sessions in both government and NGO facilities, in half of the pretest counselling session observed in government facilities individual risk assessment was not discussed with the client. This is a key aspect of the national HCT guidelines and failure to adequately address it may mean that sessions do not effect necessary behaviour change. Some counsellors focused their counselling sessions on the testing 
pro- cess and mentioned very little or nothing about HIV transmission and prevention, this was com-mon in circumstances where clients underwent

a group information session where they are normally discussed and therefore not repeated during individual counselling sessions.

The length of the counselling session is as important as its quality to ensure that clients are given time to absorb their results and plan a way forward (Magongo et al. 2002). The post-test counselling sessions observed in this study were very short for both HIV negative and positive results. Communication of HIV test results was inadequate; this was evident in the session recordings whereby counsellors were making ambiguous statements when providing results to clients whilst some counsellors were making clients read and interpret their own results. Furthermore, key aspects such as disclosure, partner referral, identification and linkage to care, support and medical services and risk reduction were not discussed adequately with clients de- spite their importance. Disclosure of HIV status and partner referral are important in establishing social support and helping prevent transmission of the virus to sexual partners as well as enabling both patients and partners to access appropriate HIV services (Chersich and Temmer- man 2008). The main aim of HCT services is to ensure clients testing HIV positive are linked care and treatment services, however a study conducted in Uganda by Wanyenze et al. (2013) has suggested an enhanced linkage approach that has significantly reduced ART initiation time. As opposed to merely providing information about nearby support services this approach includes counselling about barriers to care, assisted disclosure and a scheduled appointment at an ART clinic as well as reminders by phone or home visits. The feasibility of such an approach would have to be tested once the HIV counselling quality issues in health facilities have been addressed.

Magongo et al. (2002) and Anderson and Louw-Potgieter (2012) found similar findings in assessments conducted in South Africa where- by important components of post-test counsel- ling were not discussed. Haffejee et al. (2011) suggested that counselling services were usually rushed to accommodate competing services due to lack of counselling space in some facilities as well as the large numbers of clients needing to be attended to by the counsellors. The practice of counselling can be influenced by several factors including the type of setting as well as local conditions and social attitudes (Richter et al. 1999). There is also evidence that counsellors modify rules in the course of doing their jobs due to several factors including an attempt to tailor the counselling to individual needs (Angotti 2010), however in so doing they may exclude key messages and consequently compromise the quality of service rendered to clients. Wanyenze et al. (2013) have suggested that HIV testing can be integrated into routine health care without a lengthy counselling session particularly in settings where there are shortages of health workers however it is crucial that clients that test HIV positive are provided with more intensive post-test counselling and follow-up to ensure enrolment into care and timely treatment initiation. 
Information is a powerful tool in prevention and care of HIV and is critical to the counselling process (Njeru et al. 2009). Njeru et al. (2009) found that service users lacked trust for the quality of the knowledge of HIV and AIDS amongst the providers. This finding is supported by the results from this study which show that some lay counsellors could not explain the definition of 'window period' to their clients. A similar study conducted by Ginwalla et al. (2002) also found inadequate medical knowledge about HIV and AIDS among community volunteers. Chopra et al. (2005) cited lack of knowledge and confidence among PMTCT counsellors as the possible cause for the poor performance.

In a few of the NGOs observed, lay counsellors used written guidelines or checklists during counselling to ensure that they covered all the important aspects of counselling. A similar practice was observed among nurse counsellors in studies conducted by Ginwalla et al. (2002) and Wong (2005). This finding suggests a higher degree of standardization amongst the NGOs. Similar practices are recommended for implementation in government health facilities to ensure consistency in counselling content. The counselling sessions observed in NGO facilities covered many of the key aspects for both pre-test and post-test counselling as prescribed by the HCT guidelines; hence the content of counsel- ling was better in NGO facilities compared to government facilities. However, there were no statistically significant differences for most of the variables analysed by facility type.

It was evident from the session recordings that the counselling sessions followed a pre- dominantly educative rather than an interactive approach. Counsellors were informing clients to use condoms instead of interactively exploring various HIV prevention strategies with the client. These types of hierarchical instructions from health workers have been shown to have little effect on sexual behaviour change (Chersich and Temmerman 2008). Furthermore, it is suggested that only through effective interpersonal communication can people absorb new information meaning that people need psychosocial support and emotional counselling in addition to basic information about HIV (Haffejee et al. 2011).There was a general lack of basic counselling skills such as questioning, summarising and reflection in all the counselling sessions observed in this study and require more attention during training.

Despite confidentiality having been dis- cussed during the majority of sessions in both government and NGO facilities, in this study however it was compromised by interruptions by other staff members during some of the sessions. This key aspect has been highly rated as important by clients (Wong 2005; Njeru et al. 2009). Failure to ensure privacy affects confidentiality and may force clients to withhold important information such as HIV risk during the session. Similar findings were observed in a study by Wong (2005); counselling rooms lacked privacy and there were frequent interruptions during counselling sessions. Haffejee et al. (2011) also found that due to inadequate facilities for conducting counselling; confidentiality was compromised. 
The South African Department of Health should therefore address structural factors that hamper the provision of HCT services and thereby compromise the quality of services; this includes the provision of additional counselling spaces set aside specifically for that purpose.

Caution should be taken when interpreting the results of this study due to certain limitations. The presence of an observer during the counselling session may have prompted performance bias. To minimise this effect, care was taken for the observer to be unobtrusive and to sit-in through multiple sessions particularly by different counsellors in each HCT site. However, these findings may still be reflective of best counselling practices. The fact that observers were trained in counselling and they followed a written checklist composed of minimum standards of HIV counselling derived from the HCT guidelines, excludes the possibility of observer bias from the process. Generalizability may be limited due to the fact that sites were selected purposively to ensure diversity in terms of set- ting, model and approach and therefore may not be representative of HCT sites in South Africa. In addition, one province was excluded from the study because permission was not granted.

\section{Conclusion}

The results of this assessment illustrate that counselling does take place prior and post HIV testing, however there is enough evidence to show that the quality of counselling does not match that dictated by the National Department of Health's HCT policy guidelines. This study found that important aspects pertaining to pre and post-test counselling were not discussed adequately during sessions and this clearly indicates that the quality of counselling is com- promised and could have serious implications for the effectiveness of the HCT programme. The capacity of lay counsellors as well as structural factors are major contributors to the lack of quality of HIV counselling services in health facilities as highlighted in this study. The study indicated that the training and mentoring of counsellors needs to be addressed in order to make the HCT programme more effective. A concerning finding is in relation to the HIV/AIDS knowledge of counsellors, in this study it was evident from the session observations that counsellors did not have current information about HIV/ AIDS and that their counselling sessions were mostly unstructured. The implication is for more thorough education and knowledge components in training programs as well as the inclusion of regular knowledge updates. In a field with new information emerging at a rapid pace, there is a need for counsellors to be well informed with current information.

\section{Recommendations}

A checklist consisting of the key aspects of the counselling process will be useful for all counsellors to ensure that all key points are covered during counselling sessions, additionally it can be translated into the 11 official South African languages. On going training and mentoring as well as update processes need to be implemented in order to improve and maintain the competency of counsellors and to provide them with the 
necessary support in their counselling duties. Infrastructural challenges that compromise the quality of counselling such as shortage of counselling space indicate the urgent need for government to prioritise HIV counselling and testing services to make them more effective. Additionally, this study suggests that despite the widespread availability and accessibility of HCT services in the country regular assessments of quality should be undertaken to ensure that clients' health needs are being met.

\section{Acknowledgements}

This research has been supported by the President's Emergency Plan for AIDS Relief (PEP- FAR) through the Centers for Disease Control and Prevention (CDC) under the terms of C operative Agreement Number U2G/PSooo570. 


\section{References}

Anderson TN, Louw-Potgieter J 2012. An implementation evaluation of a voluntary counselling and testing programme for the human immunodeficiency virus (HIV) and acquired immunodeficiency syndrome (AIDS): Original research. South African Journal of Industrial Psychology, 38(1): 944-954.

Angotti N 2010. Working outside of the box: How HIV counselors in sub-Saharan Africa adapt Western HIV testing norms. Social Science and Medicine, 71: 986-993.

Chersich MF, Temmerman M 2008. Increasing access to HIV testing for women by simplifying pre- and post-test counseling.Current Womens Health Re-views, 4: 172179 .

Chopra M, Doherty T, Jackson D, Ashworth A 2005. Preventing HIV transmission to children: Quality of counselling of mothers in South Africa. Acta Peadiatrica, 94: 357-363.

Cornman DH, Christie S, Sheperd LM, MacDonald S, Amico KR et al. 2011. Counsellor-delivered HIV risk reduction intervention addresses safer sex barriers of people living with HIV/AIDS in KwaZulu-Natal, South Africa. Psychology and Health, 26(12): 1623-1641.

Delva W, Mutunga L, Quaghebeur A,Temmerman M 2006. Quality and quantity of antenatal HIV counselling in a PMTCT programme in Mombasa, Ken- ya. AIDS Care, 18(3): 189-193.

Dewing S, Matthews C, Schaay N, Cloete A, Louw J, Simbayi L 2012. "Its important to take your medication everyday, okay": An evaluation of counselling by lay counsellor for ARV adherence support in the Western Cape, South Africa. AIDS Behaviour, 17(1): 203-212.

Dinku F, Andargie G 2013. Assessment of Voluntary Counseling and Testing (VCT) service quality in terms of client satisfaction: A comparative study between public and private health institutions in Addis Ababa, Ethiopia. Science Journal of Clinical Medicine, 2(1): 1-7.

Ginwalla SK, Grant AD, Day JH, Dlova TW, Macintyre S et al. 2002. Use of UNAIDS tools to evaluate HIV voluntary counselling and testing services for mineworkers in South Africa. AIDS Care, 14(5): 707- 726.

Grabbe KL, Menzies N, Taegtmeyer M, Emukule G, Angala P et al. 2010. Increasing access to HIV counseling and testing through mobile services in Kenya: Strategies, utilization and cost-effectiveness. Journal of Acquired Immune Deficiency Syndromes, 54(3): 317-323. 
Haffejee S, Groenevald I, Fine D, Paterl R, Bowman B2010. An assessment of counselling and support services for people living with HIV in Gauteng, South Africa: Findings of a baseline study. African Journal of AIDS Research, 9(4): 367-372.

Haraka F, Mohamed A, Kilonzo G, Shao H 2012. Fac- tors affecting HIV counselling and testing among adults in Muheza District, Tanzania. Tanzania Journal of Health Research, 14(1): 1-5.

Kaaya SF, Blander J, Antelman G, Cyprian F, Emmons K et al. 2013. Randomised controlled trial evaluating the effects of an interactive group counselling intervention for HIV positive women on pre-natal depression and disclosure of HIV status. AIDS Care, 25(7): 854-862.

Kipitu U 2005. Quality Care of Voluntary Counseling and Testing: Clients' Experience, Expectations and Satisfactions in Mbeya Tanzania. Tanzania: Mbeya District Council.

MacCarthy S, Rasanathan JJK, Dourado I, Gruskin S 2014. Quality, not just quantity: Lessons learned from HIV testing in Salvador, Brazil. Global Public Health: An International Journal for Research, Policy and Practice, 9(6): 723-739.

Magongo B, Magwaza S, Mathambo V, Makhanya N 2002. National Report on the Assessment of the Public Sector's Voluntary Counselling and Testing Programme. Durban: Health Systems Trust.

Miles MB, Huberman AM 1994. Qualitative Data Analysis: An Expanded Source Book. 2nd Edition. California: Sage.

Mpiina D, Tabebwakushaba E, Mkabayi E, Luzze C, Birungi J 2013. Major barriers to condom use among client receiving counselling on sexually transmitted diseases (STI's) prevention - The AIDS Support Organisation (TASO) operational research finding a national NGO in Uganda. Sexually Transmitted Infections, 89: A289.

Mwamburi D, Dladla N, Qwana E, Lurie MN 2005. Factors associated with wanting to know HIV results in South Africa. AIDS Patient Care and STDs, 19(8): 518-525.

National Department of Health 2010. National HIV Counselling and Testing (HCT) Policy Guidelines. Pretoria: Department of Health.

Njeru MK, Blystad A, Nyamongo IK, Fylkesnes K 2009. A critical assessment of the WHO responsiveness tool: Lessons from voluntary HIV testing and counselling services in Kenya. BMC Health Services Re-search, 9(1): 243-254. 
Njeru MK, Blystad A, Shayo EH, Nyamongo IK, Fylkesnes K 2011. Practicing provider-initiated HIV testing in high prevalence settings: Consent, concerns and missed preventive opportunities. BMC Health Services Research, 11(87): 1-14.

Richards KAM, Marquez J 2005. Experiences of HIV/ AIDS counselors in Zimbabwe and their perceptions on the state of HIV/AIDS counseling in Zimbabwe. International Journal for the Advancement of Counselling, 27(3): 413-429.

Richter L, Durrheim K, Griesel D, Solomon V, Van Rooyen H 1999. Evaluation of HIV/AIDS Counselling in South Africa. Pretoria: Department of Health.

Setswe, G 2009. Models of HIV Counselling and Test- ing in the SADC Region. Paper presented at the HIV Counselling and Testing Workshop, Durban, 26 March.

Sherr L, Lopman B, Kakowa M, Dubeb S, Chawira G et al. 2007. Voluntary counselling and testing: Up- take, impact on sexual behaviour, and HIV incidence in a rural Zimbabwean cohort. AIDS, 21: 851- 860 .

Shisana O, Rehle T, Simbayi LC, Zuma K, Jooste S et al. 2009. South African National HIV Prevalence, Incidence, Behaviour and Communication Survey 2008: A Turning Tide Among Teenagers? Cape Town: HSRC Press.

Shisana O, Rehle T, Simbayi LC, Zuma K, Jooste S et al. 2014. South African National HIV Prevalence, Incidence and Behaviour Survey. Cape Town: HSRC Press.

Strode A, Van Rooyen H, Heywood M, Karim QA 2005. Scaling up HIV testing in resource-constrained settings: debates on the role of VCT and routine 'opt-in or optout' HIV testing. Southern African Journal of HIV Medicine, 6(3): 45-48.

Van Rooyen H, Richter L, Coates TJ, Boettiger M 2010. Approaches to HIV testing and counselling: Strengths and weaknesses and challenges for the way forward. In: P Rohleder, L Swartz, SC Kalichman, LC Simbayi (Eds.): HIV/AIDS in South Africa 25 Years On: Psychological Perspectives. New York: Springer, pp. 165-182.

Wanyenze, RK, Nawavvu, C, Namale, AS, Mayanja, B, Bunnell R et al. 2008. Acceptability of routine HIV counselling and testing, and HIV seroprevalence in Ugandan hospitals. Bulletin of the World Health Organization, 86: 302-309.

Wanyenze RK, Kamya M, Fatch R, Mayanja-Kizza H, Bayeewo S et al. 2013. Abbreviated HIV counselling and testing and enhanced referral to care in Uganda: A factorial randomized controlled trial. Lancet Global Health; 1: e137-145. 
WHO 2008. Acceptability of Routine HIV Counselling and Testing, and HIV Seroprevalence in Ugandan Hospitals. In: RK Wanyenze, C Nawavvu, AS Na- male, B Mayanja, R Bunnell et al. (Eds.): Bulletin of the World Health Organization, 86: 302-309.

WHO 2011. Guide For Monitoring and Evaluating National HIV Testing and Counselling (HTC) Programmes: Field-Test Version. NLM Classification: WC 503.1. ISBN978 9241501347

WHO 2012. Service Delivery Approaches to HIV Testing and Counselling (HTC): A Strategic Programme Framework. NLM Classification: WC 503.1. ISBN 978-9241593877

Wong JV 2005. A Descriptive Study of Voluntary Counselling and Testing and Rapid HIV Testing in the Accra Metro District, Ghana. Master's Thesis, Unpublished. Berlin: University of Berlin.

Zachariaha R, Ford N, Philips M, Lynch S, Massaquoid M et al. 2009. Task shifting in HIV/AIDS: Opportunities, challenges and proposed actions for sube Royal Society of Tropical Medicine and Hygiene, 103: 549-558. 